Research Article

\title{
Identification of Aircraft Wake Vortex Based on SVM
}

\author{
Weijun Pan, Zhengyuan Wu $(\mathbb{D}$, and Xiaolei Zhang $(\mathbb{D}$ \\ College of Air Traffic Management, Civil Aviation Flight University of China, Deyang, China \\ Correspondence should be addressed to Zhengyuan Wu; icy_wu@foxmail.com and Xiaolei Zhang; bmezhang@vip.163.com
}

Received 15 January 2020; Revised 27 March 2020; Accepted 22 April 2020; Published 12 May 2020

Academic Editor: Luciano Mescia

Copyright $\odot 2020$ Weijun Pan et al. This is an open access article distributed under the Creative Commons Attribution License, which permits unrestricted use, distribution, and reproduction in any medium, provided the original work is properly cited.

\begin{abstract}
The aircraft wake vortex has important influence on the operation of the airspace utilization ratio. Particularly, the identification of aircraft wake vortex using the pulsed Doppler lidar characteristics provides a new knowledge of wake turbulence separation standards. This paper develops an efficient pattern recognition-based method for identifying the aircraft wake vortex measured with the pulsed Doppler lidar. The proposed method is outlined in two stages. (i) First, a classification model based on support vector machine (SVM) is introduced to extract the radial velocity features in the wind fields by combining the environmental parameters. (ii) Then, grid search and cross-validation based on soft margin SVM with kernel tricks are employed to identify the aircraft wake vortex, using the test dataset. The dataset includes wake vortices of various aircrafts collected at the Chengdu Shuangliu International Airport from Aug 16, 2018, to Oct 10, 2018. The experimental results on dataset show that the proposed method can identify the aircraft wake vortex with only a small loss, which ensures the satisfactory robustness in detection performance.
\end{abstract}

\section{Introduction}

Aircraft wake turbulence is a function of an aircraft producing lift, which is invisible and harmful to the trailing aircraft, especially, hazardous in the takeoff or landing phases of the flight [1]. The study on the aircraft wake vortex can help in regulating the wake turbulence separation standards to ensure flight safety and avoid wake encounters. Thus, the conservative wake turbulence separation is the main factor that influences the increase of airspace capacity in the limited capacity of airspace.

Computational fluid dynamics (CFD) and field measurements are two important methods to study the behavior of the aircraft wake vortex. The CFD supports the consistent investigation of wake vortex behavior under various environmental conditions but has not yet a significant impact on aircraft spacing for airport capacity enhancement [2]. The high accuracy and resolution of the lidar make it the most effective device at field measurement. Particularly, the new wake turbulence separation standards mainly rely on lidar measurements and technologies, which have emerged as an efficient tool for predicting and characterizing the aircraft wake vortex with different environmental parameters in different flight phases [3-9].

However, the field measurement dataset collected by the lidar cannot be used directly because it contains nonwake wind field data. In addition, the dataset does not match with the correct flight because the relative position of the aircraft and the lidar is difficult to measure. On the contrary, the volume of data collected by the lidar is very large and it takes a long time to be processed manually even with the automatic dependent surveillance-broadcast (ADS-B) or quick access recorder (QAR) data. Particularly, the lower quality data caused by the sensor scanning and environmental parameters make the traditional manual methods incapable of working satisfactorily for the identification of large number of wake vortex data with high accuracy. Therefore, a fast and efficient method is required to automatically annotate the lidar data.

Machine learning maybe a powerful method to classify the lidar data into nonwake and wake data by generating images from lidar data, but its performance relies on the visualization of lidar data and the configurations of color maps for different velocity. Unsuitable color maps may lead 
to low performance and loss of information in the process of data to images.

In this paper, a classification model based on support vector machine (SVM) is proposed for automatically identifying the lidar data. The remainder of this paper is organized as follows. Section 2 details our dataset, which was collected at the Chengdu Shuangliu International Airport (CSIA) from Aug 16, 2018, to Oct 10, 2018. The proposed method is described in Section 3. The results are presented in Section 4 followed by conclusion in Section 5 .

\section{Data Sources}

The aircraft wake vortex behaves like large rolling air masses behind the flight path of the aircraft, and the main motion direction is perpendicular to the flight path of the aircraft in the plane. Thus, the lidar observational transverse mode is the range height indicator (RHI), as shown in Figure 1.

The mode of the lidar used for the experiment is Wind3D 6000, which is manufactured by Leica-Lidar Transient Technology Ltdlenleadertwodots. In addition, this lidar is based on all-fiber laser technology and the heterodyne detection technology, as shown in Figure 2(a). The volume of the lidar is small, and its spatial and temporal resolutions are high enough for the detection of the aircraft wake vortex. The specifications of Wind3d 6000 are listed in Table 1.

The field measurement is achieved at the Chengdu Shuangliu International Airport from Aug 16, 2018, to Oct 10, 2018. The position of the lidar is at point $A$, and the threshold of runway $02 \mathrm{R}$ is at point $B$, as shown in Figure $2(\mathrm{~b})$. The elongation line of the runway centerline and the detection plane of the RHI mode intersect at point $C$. The distance from the lidar to the cross-point $C$ is 503 meters, and the distance between the threshold and cross-point $C$ is 1468 meters.

The specific configuration of field measurement is summarized in Table 2. The runway $02 \mathrm{R}$ is for landing. For the aircraft following the standard approaching procedure, the altitude of the landing aircraft at point $C$ is 78 meters, which can be covered by the scan range of the lidar. The scanning rate is $1^{\circ} / \mathrm{s}$, and the elevation ranges from $0^{\circ}$ to $10^{\circ}$. Thus, the scan duration is about 10 seconds, meeting the demands for wake vortex investigation.

A day with good weather was selected to avoid the influence of rain and fog. The dataset contained 344 samples in one day.

\section{Methodology}

The lidar can only measure the velocity at the direction of the line-of-sight (LOS) because of the limitation of the principle of the Doppler effect. The LOS velocity also called radial velocity is discrete, so the set of the radial velocity of the wind field is defined as $V_{r}$ :

$$
V_{r}=\left\{v_{r}\left(r_{i}, \theta_{j}\right)\right\}, \quad i \in[1, n], j \in[1, m],
$$

where $r_{i}$ is the $i$-th range which means the distance from the lidar, $\theta_{j}$ is $j$-th angle which denotes the pitch angle of the lidar, and $v_{r}\left(r_{i}, \theta_{j}\right)$ indicates the radial velocity of the point at coordinate $\left(r_{i}, \theta_{j}\right)$.
The sample data of Airbus 320 are visualized in Figure 3, where the color bar represents the velocity. It should be noted that the area of the wake vortex colored with a pair red and green is only a small part of the entire detected area.

\subsection{Feature Extraction}

3.1.1. Velocity Envelop at Different Angle or Range. The envelope of radial velocity $v_{r}$ is the main feature when the aircraft produces the wake vortices at the light wind. $D_{r}\left(r_{i}\right)$ and $D_{a}\left(\theta_{j}\right)$ are introduced as follows:

$$
\begin{array}{ll}
D_{r}\left(r_{i}\right)=\max \left(v_{r}\left(r_{i}, \theta_{j}\right)\right)-\min \left(v_{r}\left(r_{i}, \theta_{j}\right)\right), & \forall j \in[1, m], \\
D_{a}\left(\theta_{j}\right)=\max \left(v_{r}\left(r_{i}, \theta_{j}\right)\right)-\min \left(v_{r}\left(r_{i}, \theta_{j}\right)\right), & \forall i \in[1, n],
\end{array}
$$

where $D_{r}\left(r_{i}\right)$ and $D_{a}\left(\theta_{j}\right)$ are the max difference radial velocities at each range $r_{i}$ and each angle $\theta_{j}$, respectively. Then, the core position of the vortices can be determined in terms of the difference between the maxima (for the positive envelope) and the minima (for the negative envelope).

3.1.2. Velocity Difference of Rectangular Area. The envelopes in Section 3.1.1 only consider the difference of maximum positive and negative velocities which may not be the true area of the aircraft wake vortices due to the existence of the nonuniform background wind field. As shown in Figure 4, the area colored red as the maximum velocity difference at the range about 675 meters does not contain the wake vortices. Actually, the most significant feature of the aircraft wake vortices is the pair of positive and negative velocities, which is located in a rectangular area. Thus, $D_{\text {rec }}$ is defined to express this feature:

$$
D_{\mathrm{rec}}\left(r_{i}, \theta_{j}\right)=\sum\left(v_{r, \mathrm{UL}}+v_{r, \mathrm{LR}}\right)-\sum\left(v_{r, \mathrm{UR}}+v_{r, \mathrm{LL}}\right),
$$

where $v_{r, \mathrm{UL}}, v_{r, \mathrm{UR}}, v_{r, \mathrm{LL}}$, and $v_{r, \mathrm{LR}}$ are the average radial velocities of upper-left, upper-right, lower-left, and lowerright areas, respectively, as shown in Figure 5. The specific size of the total area is 120 meters in width and 60 meters in height, and the area is divided into four same size subareas, which can cover most of the wake vortices.

3.2. Environmental Parameters. The aircraft wake vortices are affected by various factors, such as temperature, humidity, wind shear, and turbulence. In order to increase the accuracy and robustness of the model, the temperature $T$, the average velocity $v_{\mathrm{wV}}$, and the background wind field direction $\theta_{\mathrm{wd}}$ and the air pressure $P$ are considered. The data are collected by the automatic meteorological station of CAAC Southwest Air Traffic Management Bureau, Chengdu, with a data acquisition rate of $1 \mathrm{~Hz}$.

3.3. Support Vector Machine. Support vector machine (SVM) is a powerful machine learning method that finds the hyperplane to classify the given vectors. The vector defined in Section 3.1 and 3.2 is 


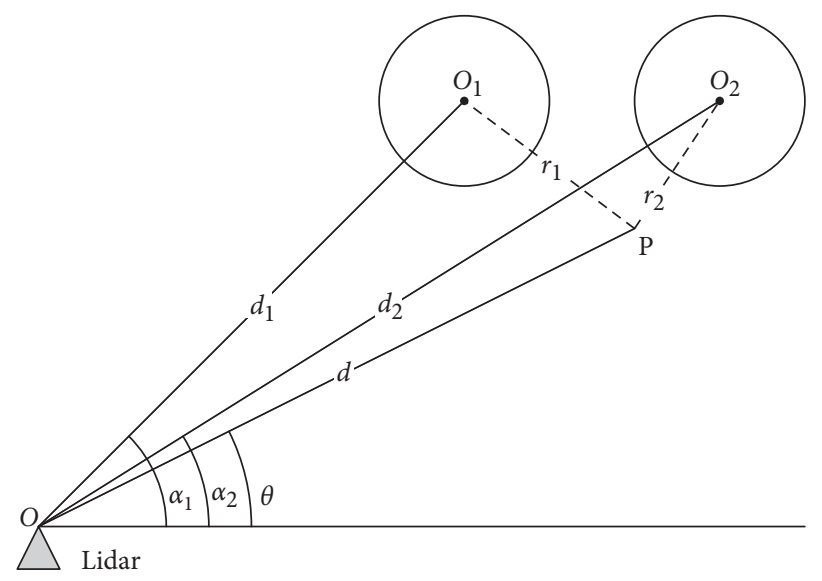

Figure 1: The observation mode of lidar, RHI.

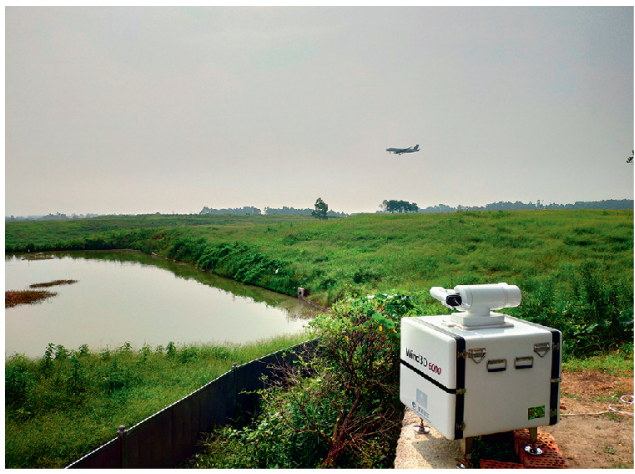

(a)

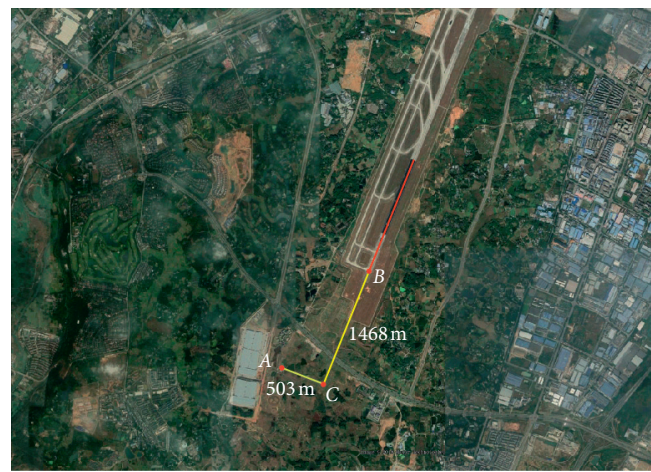

(b)

Figure 2: (a) Wind3D 6000. (b) Sketch map of field measurement.

TABLE 1: The specifications of Wind3D 6000.

\begin{tabular}{lc}
\hline Qualification & Specifications \\
\hline Wavelength $(\mu \mathrm{m})$ & 1.55 \\
Pulse repetition $(\mathrm{kHz})$ & 10 \\
Sampling rate $(\mathrm{GHz})$ & 1 \\
Data update rate $(\mathrm{Hz})$ & 4 \\
Pulse width $(\mathrm{ns})$ & $100-400$ \\
Pulse energy $(\mu \mathrm{J})$ & 150 \\
Measurement range $(\mathrm{m})$ & $45-6000$ \\
Range resolution $(\mathrm{m})$ & $15-50$ \\
\hline
\end{tabular}

TABLE 2: Experimental configuration for the RHI scanning mode.

\begin{tabular}{lc}
\hline Parameters (unit) & Value \\
\hline Azimuth angle $\left({ }^{\circ}\right)$ & 112 \\
Scanning rate $(\% / \mathrm{s})$ & 1 \\
Elevation range $\left({ }^{\circ}\right)$ & $0-10$ \\
Elevation angle resolution $\left({ }^{\circ}\right)$ & $0.2 \pm 0.03$ \\
Detection radial range $(\mathrm{m})$ & $45-885$ \\
Longitudinal resolution $(\mathrm{m})$ & 15 \\
Distance between point $A$ and $B(\mathrm{~m})$ & 503 \\
Distance between point $B$ and $C(\mathrm{~m})$ & 1468 \\
\hline
\end{tabular}

$$
\begin{aligned}
& \mathbf{x}_{i}=\left(D_{r}, D_{a}, D_{\mathrm{rec}}, T, v_{\mathrm{wv}}, \theta_{\mathrm{wd}}, P\right), \\
& y_{i} \in\{-1,+1\},
\end{aligned}
$$

where $i \in[1, N], y_{i}=-1$ denotes no wake vortex, and $y_{i}=+1$ is the wake vortex.

It becomes a typical classification problem with SVM: finding the maximum-margin hyperplane $[10,11]$ :

$$
\begin{array}{ll}
\underset{\mathbf{w}, b, \xi}{\arg \min } & \frac{1}{2} \mathbf{w}^{T} \mathbf{w}+C \sum_{i=1}^{l} \xi_{i} \\
\text { s.t. } & y_{i}\left(\mathbf{w}^{T} x_{i}+b\right) \geq 1-\xi_{i}, \\
& \\
& \xi_{i} \geq 0 .
\end{array}
$$

Because feature vector $\left(\mathbf{x}_{1}, y_{1}\right),\left(\mathbf{x}_{2}, y_{2}\right), \ldots,\left(\mathbf{x}_{n}, y_{n}\right)$ is not linearly separable, the vectors are mapped into a higher dimensional space by kernel function $\phi$ : 


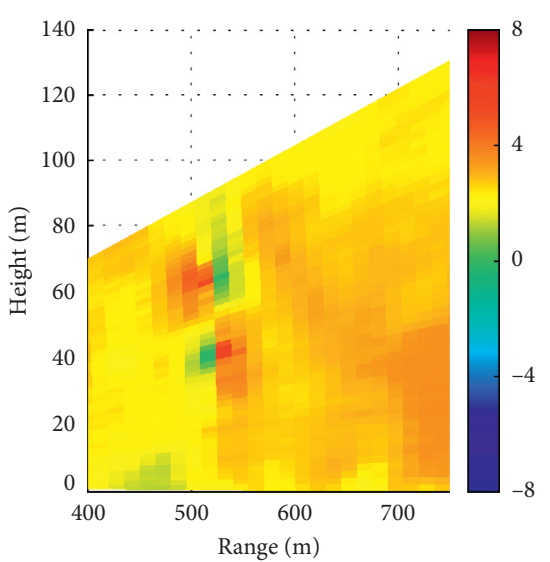

(a)

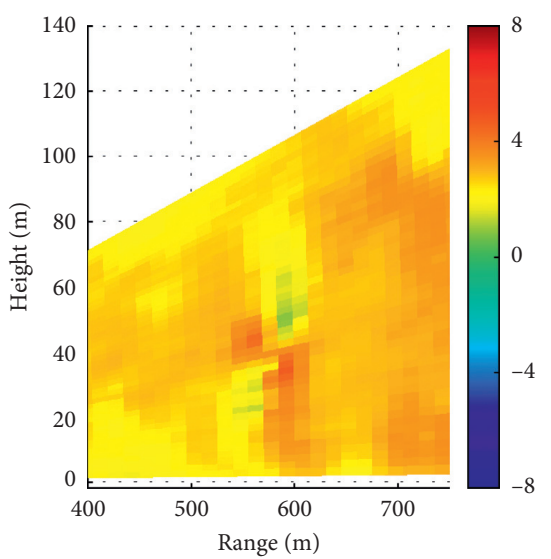

(b)

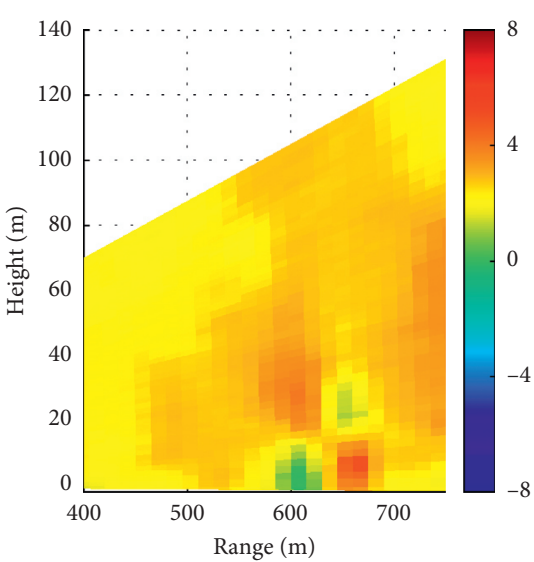

(c)

FIgURE 3: Visualization of lidar data for Airbus-A320.

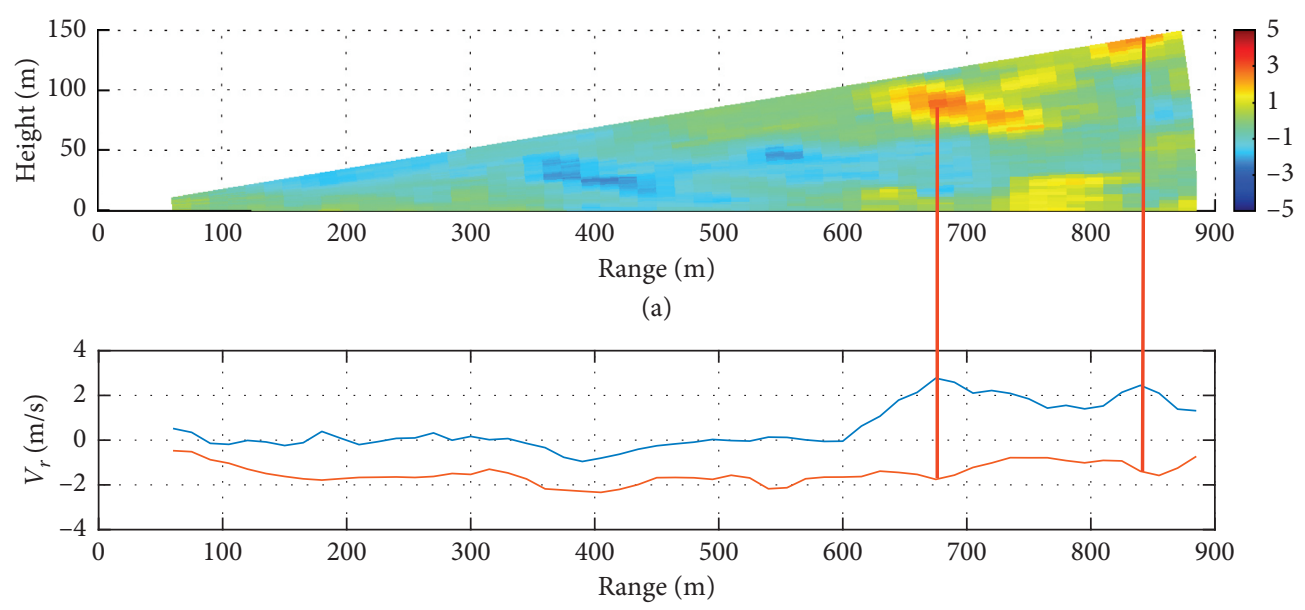

- Maximum positive speed

— Maximum negative speed

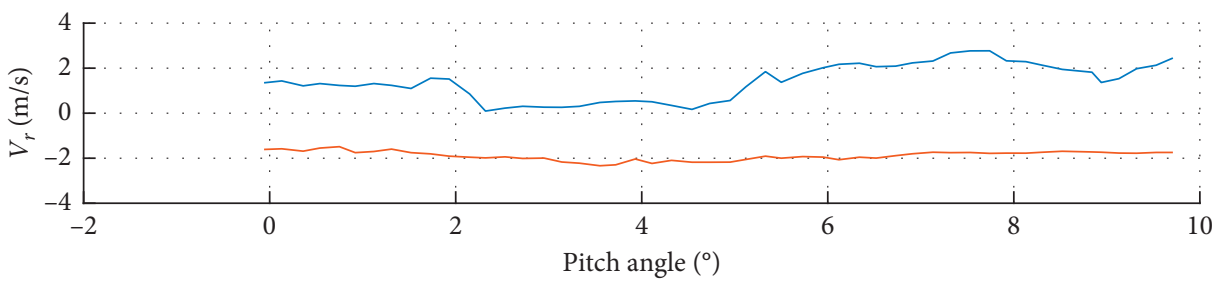

_ Maximum positive speed

Maximum negative speed

(c)

FIgURE 4: Example of the velocity envelopes in Airbus-A320 for the vortex circulation.

$$
\begin{array}{ll}
\underset{\mathbf{w}, b, \xi}{\arg \min } & \frac{1}{2} \mathbf{w}^{T} \mathbf{w}+C \sum_{i=1}^{l} \xi_{i} \\
\text { s.t. } & y_{i}\left(\mathbf{w}^{T} \phi\left(x_{i}\right)+b\right) \geq 1-\xi_{i}, \\
& \xi_{i} \geq 0
\end{array}
$$

where $C$ is the penalty parameter of the error term, $\phi$ is the function that maps the training vector $\mathbf{x}_{i}$ into a higher dimensional space, $\xi$ is the linear constraint, and $\mathbf{w} \phi\left(x_{i}\right)+b=0$ is the hyperplane.

The number of features is 7 , which is less than the number of vectors in the dataset. Thus, the radial basis function (RBF) is chosen as the kernel function to reduce the complexity: 


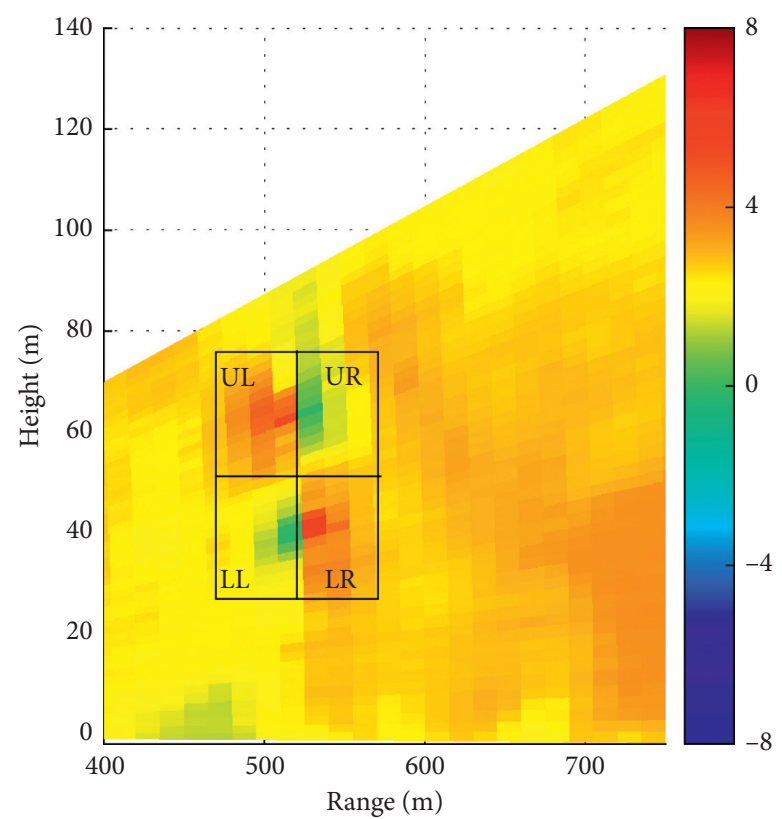

FIGURE 5: Wake vortex regions in the detection area.

$$
K\left(\mathbf{x}_{i}, \mathbf{x}_{j}\right)=e^{-\gamma\|\mathbf{x}-\mathbf{y}\|},
$$

where $\gamma$ is the parameter of RBF kernel and is equal to $1 / 2 \sigma^{2}$.

The dimension of feature increases with the number of samples in the data set after choosing the RBF kernel and $\phi\left(\mathbf{x}_{\mathbf{j}}\right)$ :

$$
\phi\left(\mathbf{x}_{i}\right)=\left(e^{-\gamma\left\|\mathbf{x}_{i}-\mathbf{x}_{1}\right\|}, e^{-\gamma\left\|\mathbf{x}_{i}-\mathbf{x}_{2}\right\|}, \ldots, e^{-\gamma\left\|\mathbf{x}_{i}-\mathbf{x}_{m}\right\|}\right) .
$$

3.4. Algorithm for Wake Vortex Identification. The entire procedure for wake vortex identification is illustrated by the flowchart in Figure 6.

\section{Experiment and Results}

4.1. General Setting. In order to avoid the range of different features, the attributes are linearly scaled in the range $[0,1]$. The values of features in the vector are represented as real numbers in SVM because the performance is significantly influenced if the attributes in greater numeric ranges dominate those in smaller numeric ranges [12]. Therefore, after the features are extracted from the radial velocity of the wind field, all the attributes are linearly scaling into $[0,1]$, as shown in Table 3.

4.2. Model Training. The two parameters $C$ and $\gamma$ of RBF kernel affect the performance of SVM. In order to achieve the best performance, grid search is performed on $C$ and $\gamma$, which is the most used method in SVM. The tool used for solving the SVM is the scikit-learn $[13,14]$ package in Python based on LIBSVM. The cross-validation is the method used to evaluate the performance of the model with different $C$ and $\gamma$. The train dataset is divided into 5 folds. The first range of the grid search is $C=2^{-5}, 2^{-4}, \ldots, 2^{14}, 2^{15}$

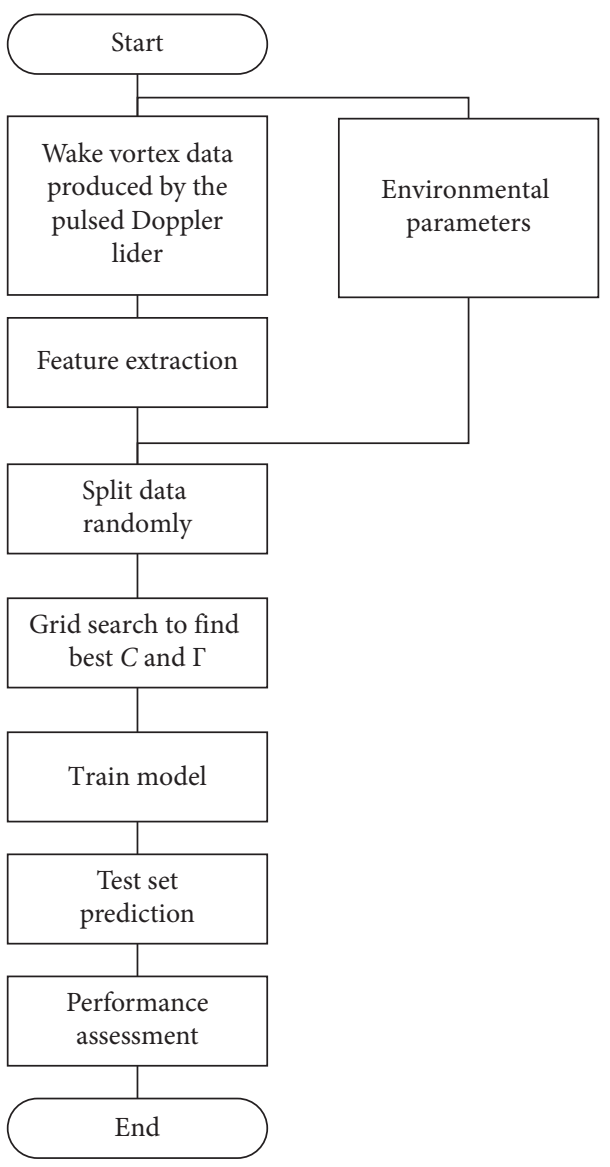

FIgURE 6: Flowchart of the proposed wake vortex finding algorithm.

and $\gamma=2^{-15}, 2^{-14}, \ldots, 2^{2}, 2^{3}$, the cross-validation score of the 1st time grid search is shown in Figure $7(\mathrm{a})$, and $C=$ 4096.00 and $\gamma=1.00$ are the best parameters with the score 
TABLE 3: Range of attributes.

\begin{tabular}{lcc}
\hline Attributes (unit) & Range & Average \\
\hline$D_{r}(\mathrm{~m} / \mathrm{s})$ & $1.51-47.21$ & 4.72 \\
$D_{a}(\mathrm{~m} / \mathrm{s})$ & $0.00-31.66$ & 4.59 \\
$D_{\mathrm{rec}}(\mathrm{m} / \mathrm{s})$ & $0.00-13.30$ & 1.44 \\
$T\left({ }^{\circ} \mathrm{C}\right)$ & $25.10-29.10$ & 27.32 \\
$v_{\mathrm{wv}}(\mathrm{m} / \mathrm{s})$ & $0.14-4.13$ & 2.63 \\
$\theta_{\mathrm{wd}}\left({ }^{\circ}\right)$ & $57.00-359.00$ & - \\
$P(\mathrm{hPa})$ & $1005.51-1008.37$ & 1006.74 \\
\hline
\end{tabular}

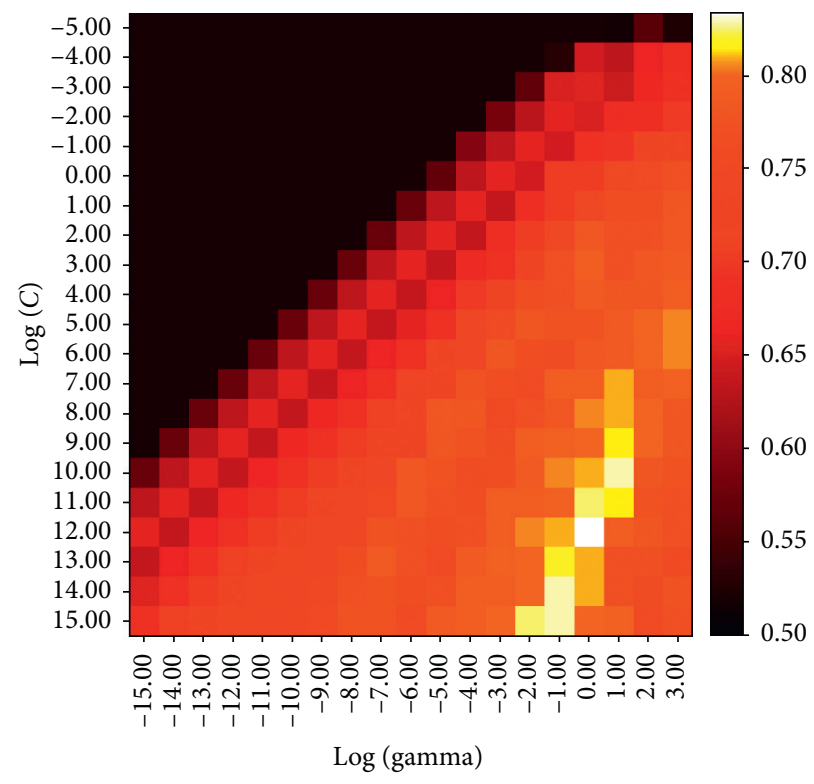

(a)

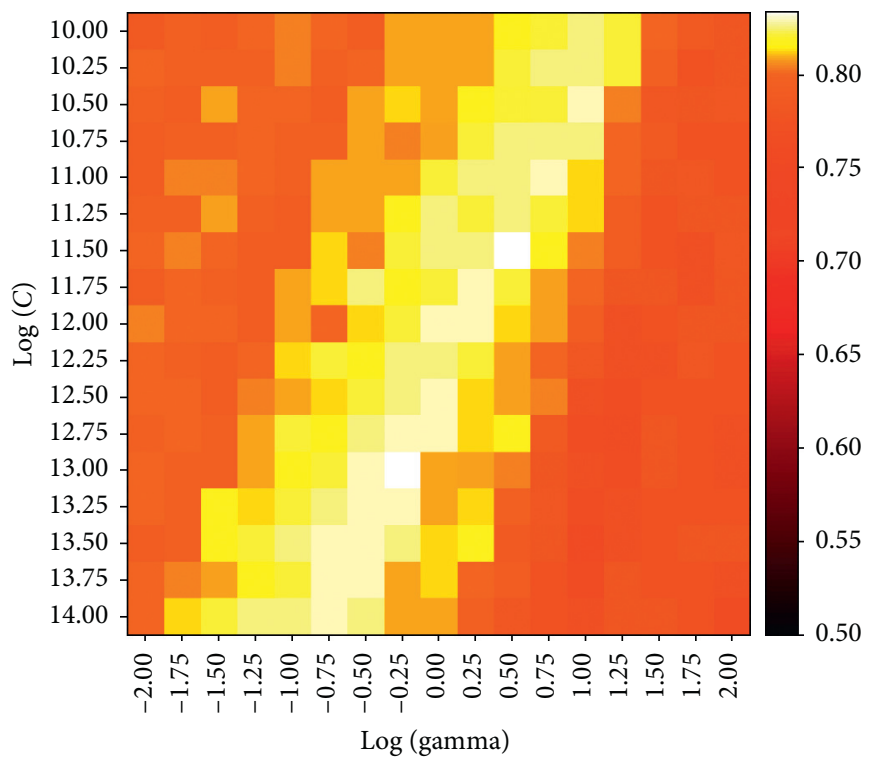

(b)

Figure 7: (a) Score of the 1st time grid search. (b) Score of the 2nd time grid search.

of 0.8296 . The grid search is performed again in the range $C=2^{10}, 2^{10.25}, \ldots, 2^{13.75}, 2^{14}$ and $\gamma=2^{-2}, 2^{-1.75}, \ldots, 2^{1.75}, 2^{2}$, and the score increases to 0.8333 when $C=2896.31$ and $\gamma=$ 1.41 at this time. The results are shown in Figure $7(\mathrm{~b})$.

4.3. Performance Assessment. In order to test the feasibility of the proposed method in aircraft wake vortex identification, the performance was evaluated by measures of F1score, receiver operating characteristic (ROC) curves [15], average conditional probability (ACP), and approximate correlation (AC). The F1-score conveys the balance between the precision and the recall. The ROC curves explore the effects on the true positive rate (TPR) and the false positive rate (FPR) as the position of an arbitrary decision threshold is varied, and the area under the ROC curve (AUC) with a larger value indicates more accurate identification. The ACP which ranges from 0 to 1 and the $\mathrm{AC}$ which ranges from -1 to 1 are used as the measures of overall identification accuracy.

4.4. Results. The performance of the final model with $C=$ 2896.31 and $\gamma=1.41$ is shown in Table 4. The number of support vectors is 42 among the 240 samples in the training set. To the best of the authors' knowledge, this may be the first attempt to use machine leaning strategies in the field of wake vortex identification. In Pan et al.'s [16] method, the authors have used the k-nearest neighbor (KNN) to characterize the different features between wake vortices and background through the distance metric. To evaluate the validity of the proposed identification model, a comparison with the KNN method is conducted using the same dataset.

The performance of the test set predicted by KNN and SVM, which are trained using the same training set, is shown in Table 5. It can be observed that the proposed method provides a consistently higher identification rate on the dataset than the KNN. Figure 8(a) is the plot of the ACP and $\mathrm{AC}$ at different classification thresholds. Using a threshold value of 44 percent, a maximum ACP of 0.805 and a maximum AC of 0.609 are obtained. Similarly, as shown in Figure $8(\mathrm{a})$, the KNN classifier with a $k$ value of 38 provides the maximum classification accuracy of $\mathrm{ACP}=0.803$ and $\mathrm{AC}=0.607$. Clearly, the best performance is obtained with the SVM-based method. Considering the accuracy of the pulsed Doppler lidar measurements, the results of the proposed SVM-based method are sufficiently high. An additional classification experiment was designed to assess the proposed method. Figures 8(b) and 8(d) show the ROC 
TABLE 4: Confusion matrix of the SVM classifier with $C=2896.31$ and $\gamma=1.41$.

\begin{tabular}{lcccc}
\hline Dataset & True positive & False positive & False negative & True negative \\
\hline Test set & 29 & 6 & 17 & 51 \\
\hline
\end{tabular}

TABLE 5: Performance of trained KNN and SVM classifiers on the same test set [16].

\begin{tabular}{lcccc}
\hline Model & Accuracy & Precision & Recall & \\
\hline KNN & 0.77 & 0.81 & 0.63 & 0.79 \\
SVM & 0.78 & 0.83 & 0.63 & 0.80 \\
\hline
\end{tabular}

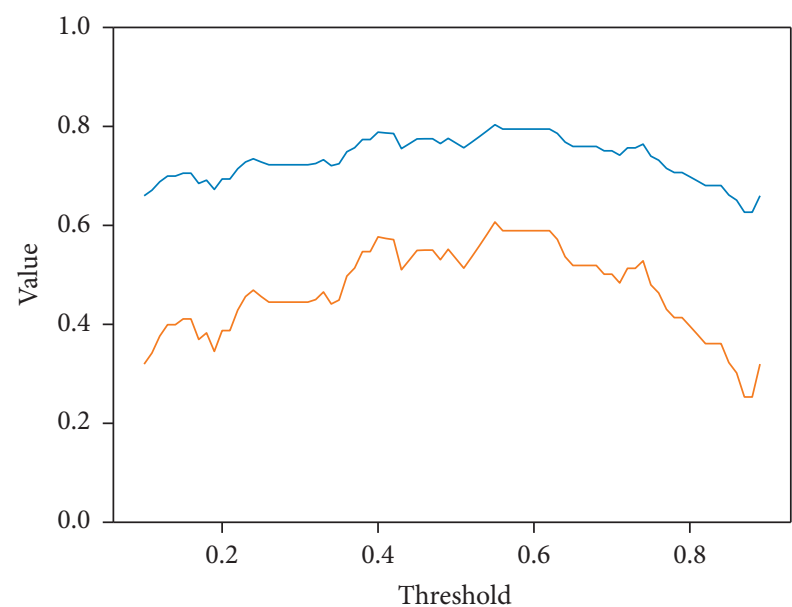

- ACP curve

- AC curve

(a)

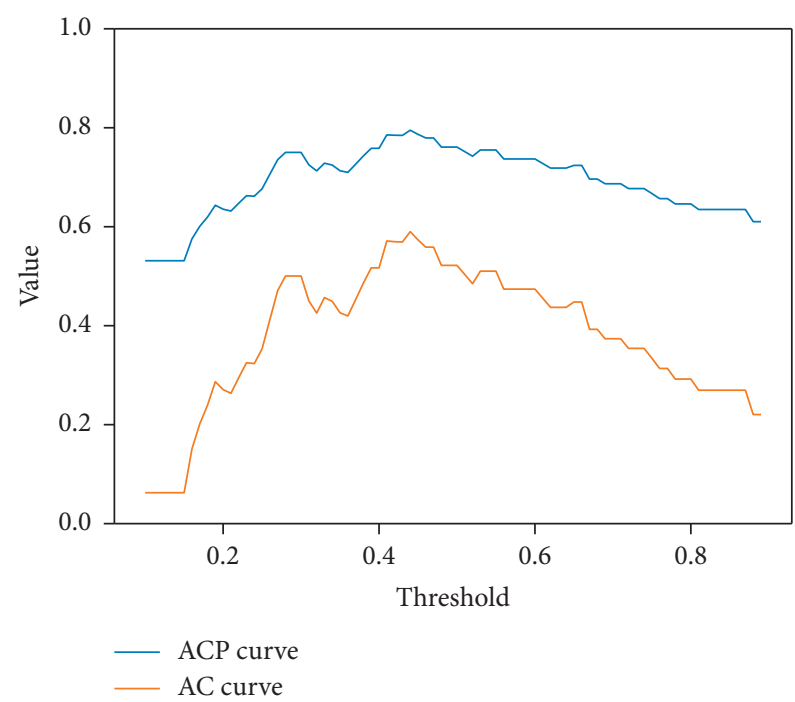

(c)

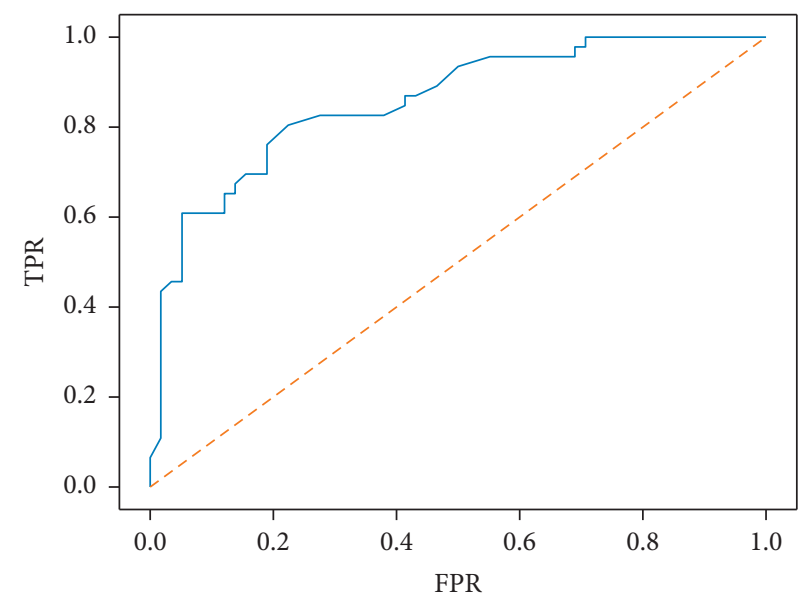

ROC curve $(\mathrm{AUC}=0.86)$

(b)

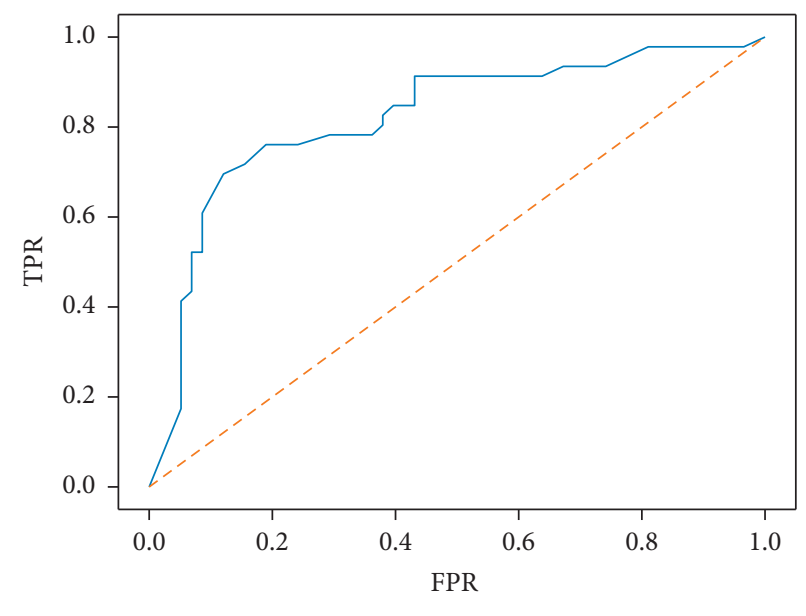

- ROC curve $(\mathrm{AUC}=0.82)$

(d)

Figure 8: AC and ROC Curves. (a) KNN AC curve. (b) KNN ROC curve. (c) SVM AC curve. (d) SVM ROC curve.

curves of the proposed method by using the dataset. It can be observed that the two methods perform well (AUC > 0.8) in the classification of wake vortices. The method based on
SVM achieves a low classification performance $(\mathrm{AUC}=0.81)$. Consider that the wake vortex data measured by using pulsed the Doppler lidar provide signal uncertainty 
and eccentricity caused by sensor scanning patterns, background winds, and a receiver's adjusted gain response. The results obtained using the proposed method are acceptable.

\section{Conclusion}

In this paper, an efficient method based on the soft margin SVM is proposed to identify the aircraft wake vortex. The method is applied to recognize the aircraft wake vortex by using the pulsed Doppler lidar characteristics acquired at the Chengdu Shuangliu International Airport from Aug 16, 2018, to Oct 10, 2018. From the measured data, the velocity envelops are first used to measure the significant feature of the aircraft wake vortex. Then, the grid search and crossvalidation based on the soft margin SVM with the RBF kernel are applied to identify the characteristics of the aircraft wake vortex. The experimental results demonstrate that the final classifier with $C=2896.31$ and $\gamma=1.41$ has the best performance in cross-validation, and the precision is more than $70 \%$ and the AUC of ROC curve is 0.81 . The results also validate that the proposed method has higher accuracy with satisfactory robustness in identifying the aircraft wake vortex. The accuracy of the aircraft wake vortex achieved by the method proposed in this paper is quite sufficient for the analysis of wake vortex behavior under wind field conditions.

\section{Data Availability}

The data used to support the findings of this study have not been made available because the raw data required to reproduce these findings cannot be shared at this time as the data also form a part of an ongoing study.

\section{Conflicts of Interest}

The authors declare that they have no conflicts of interest.

\section{Acknowledgments}

This work was supported by the National Natural Science Foundation of China (Grant no. U1733203) and the Civil Aviation Administration of China's Safety Capability Construction Program (Grant nos. TM2018-9-1/3 and TM2019-16-1/3).

\section{References}

[1] T. Gerz, F. Holzäpfel, and D. Darracq, "Commercial aircraft wake vortices," Progress in Aerospace Sciences, vol. 38, no. 3, pp. 181-208, 2002.

[2] J. N. Hallock and F. Holzäpfel, "A review of recent wake vortex research for increasing airport capacity," Progress in Aerospace Sciences, vol. 98, pp. 27-36, 2018.

[3] F. Köpp, S. Rahm, and I. Smalikho, "Characterization of aircraft wake vortices by $2-\mu \mathrm{m}$ pulsed Doppler lidar," Journal of Atmospheric and Oceanic Technology, vol. 21, no. 2, pp. 194-206, 2004.

[4] M. Harris, R. I. Young, F. Köpp, A. Dolfi, and J.-P. Cariou, "Wake vortex detection and monitoring," Aerospace Science and Technology, vol. 6, no. 5, pp. 325-331, 2002.
[5] F. Holzäpfel, T. Gerz, F. Köpp et al., "Strategies for circulation evaluation of aircraft wake vortices measured by lidar," Journal of Atmospheric and Oceanic Technology, vol. 20, no. 8, pp. 1183-1195, 2003.

[6] S. Rahm, I. Smalikho, and F. Köpp, "Characterization of aircraft wake vortices by airborne coherent Doppler lidar," Journal of Aircraft, vol. 44, no. 3, pp. 799-805, 2007.

[7] S. Rahm and I. Smalikho, "Aircraft wake vortex measurement with airborne coherent Doppler lidar," Journal of Aircraft, vol. 45 , no. 4 , pp. $1148-1155,2008$.

[8] M. Keane, D. Buckton, M. Redfern et al., "Axial detection of aircraft wake vortices using Doppler lidar," Journal of Aircraft, vol. 39 , no. 5 , pp. $850-861,2002$.

[9] F. Holzäpfel, "Probabilistic two-phase wake vortex decay and transport model," Journal of Aircraft, vol. 40, no. 2, pp. 323-331, 2003.

[10] B. E. Boser, I. M. Guyon, and V. N. Vapnik, "A training algorithm for optimal margin classifiers," in Proceedings of the Fifth Annual Workshop on Computational Learning Theory, pp. 144-152, Pittsburgh, PA, USA, July 1992.

[11] C. Cortes and V. Vapnik, "Support-vector networks," $M a-$ chine Learning, vol. 20, no. 3, pp. 273-297, 1995.

[12] C.-W. Hsu, C.-C. Chang, C.-J. Lin et al., A Practical Guide to Support Vector Classification, National Taiwan University, Taipei, Taiwan, 2003.

[13] F. Pedregosa, G. Varoquaux, A. Gramfort et al., "Scikit-learn: machine learning in Python," Journal of Machine Learning Research, vol. 12, pp. 2825-2830, 2011.

[14] L. Buitinck, G. Louppe, M. Blondel et al., "API design for machine learning software: experiences from the scikit-learn project," in ECML PKDD Workshop: Languages for Data Mining and Machine Learning, pp. 108-122, Prague, Czech Republic, September 2013.

[15] T. Fawcett, "An introduction to roc analysis," Pattern Recognition Letters, vol. 27, no. 8, pp. 861-874, 2006.

[16] W. Pan, Z. Wu, and X. Zhang, "Identification of aircraft wake vortex based on k-nearest neighbor," 2019, http://kns.cnki. net $/ \mathrm{kcms} /$ detail/51.1125.TN.20190926.1704.002.html. 